\title{
CORRELATION BETWEEN MIGRAINE SEVERITY AND COMORBIDITIES IN EPISODIC MIGRAINE PATIENTS
}

\author{
Adriana Bulboaca, Corina Ursu, Ana Uifalean, Angelo Bulboaca \\ Neurology Department, Clinical Rehabilitation Hospital, \\ University of Medicine and Pharmacy "Iuliu Hatieganu”, Cluj-Napoca
}

\begin{abstract}
Introduction. Migraine is an important cause of disability that affects the quality of life producing a substantial social impairment.

Objectives. The aim of this study was to evaluate the correlation between migraine severity and comorbidities in patients with episodic migraine.

Methods. 2 groups of patients were included in the study: group 1 - control group (healthy subjects, $n=50$ ), and group 2 - study group (patients with episodic migraine attacks, $n=65$ ). Both groups were assessed for nonmodifiable risk factors (age), and modifiable risk factors: BMI (body mass index), lipids profiles (LDL-cholesterol, HDL-cholesterol, triglycerides), inflammatory parameters (C reactive protein and fibrinogen), and endothelial dysfunction (nitric oxide synthesis expressed as NOx). The migraine was diagnosed according to ICHD-2 (International Classification of Headache Disorders) and the severity was evaluated by MIDAS (Migraine Disability Assessment Scale). The study group was compared with control group. The parameters that showed statistic significance were correlated with MIDAS scale.

Results. There was a significant correlation between the severity and frequency of migraine attacks (evaluated by MIDAS scale) and BMI. The subgroup with higher inflammatory parameters and decreased NOx had an increased MIDAS score.

Conclusions. The connection between obesity, inflammatory parameters and migraine may be useful for new prophylaxis potent therapies. The individual personalized therapies according with particular risk factors of each patient may be a chance for better results. Identification and control of risk factors in migraine patients may be particularly important.
\end{abstract}

Keywords: migraine, risk factors, obesity, inflammation

\section{INTRODUCTION}

Migraine is a common condition that requires global management aimed to reducing the frequency of the attacks, lowering the associated disability and improving the patients' quality of life. Episodic migraine and chronic migraine are the part of the same spectrum disorders, and the identification of risk factors and comorbidity profile for both clinical forms are important in order to therapeutic improvement of functional status of the patients with migraine. Because there are no biological markers for migraine, the clinical examination and exclusion of other cause of headache is the base of migraine diagnosis (1). Because episodic migraine progress to chronic migraine at the rate of $2.5 \%$ per year (2), and because the chronic migraine is associated more frequent with disability than episodic migraine (3), the identification of risk factors and comorbidities among episodic migraine patients may contribute to a better prognosis for migraine patients. The pathophysiologic mechanisms for episodic migraine transformation in chronic migraine, is under research (4). Several comorbidities are associated to migraine. Cerebrovascular disorders, both ischemic, and hemorrhagic, were associated with migraine, while the association between migraine and cardiovascular disease remains poorly defined (5). Additionally, individuals with migraine have a higher prevalence of risk factors 
known to be associated with cardiovascular disease, including hypertension, diabetes, and hyperlipidemia (6). Obesity can also be linked with migraine prevalence, frequency, and disability both in pediatric and adult subjects (7). The association between obesity and episodic migraine is strongest in those younger than 50 years and women (8). The $\mathrm{C}$ reactive protein (CRP) as a biomarker of inflammation showed to be potential associated with migraine (9). The endothelial dysfunction (expressed by nitric oxide synthesis - a biomarker for endothelial dysfunction) was also associated with migraine (10).

The aim of this study was to identify the relationship between associated disorders or comorbidities in episodic migraine patients. We sought to find if there is a correlation between blood pressure, metabolic (carbohydrate and lipid profile), inflammatory parameter, and nitro-oxidative stress with severity of migraine assessed by Migraine Disability Assessment Scale (MIDAS).

\section{MATERIAL AND METHODS}

\section{Study groups and protocol}

The study had the local Ethics Committee approval (Clinical Rehabilitation Hospital, Cluj-Napoca, Romania) and the Informed Consent for each patient. The subjects included in this study were referred to Clinical Rehabilitation Hospital, ClujNapoca, Romania for headache during March 2012 - March 2014. Two groups of patients were selected for the study: group 1 (control group - healthy subjects, $\mathrm{n}=50$ ), group 2 (study group - patients with migraine, $\mathrm{n}=65$ ). The migraine was diagnosed according to ICHD-2 (International Clasiffication of Headache Disorders) and the severity was evaluated by MIDAS (Migraine Disability Assessment Scale) (11). Patients with episodic migraine without aura were included in the study. Chronic migraine and cerebrovascular diseases patients (assessed by cranial MRI) were excluded. Other causes of headache or medication overuse headache were also excluded. Both groups were assessed for non-modifiable risk factors (age) and modifiable risk factors: BMI (body mass index), lipids profiles (LDL-cholesterol, HDL-cholesterol, triglycerides), inflammatory parameters ( $\mathrm{C}$ reactive protein and fibrinogen). The endothelial dysfunction was assessed by nitric oxide evaluation as nitrite an nitrate plasma level (NOx)(Griess reaction) (12). The smoking/non-smoking status and the alcohol consumption were evaluated by questionnaire for each patient. Significant ethylic alcohol use was considered a daily dose above $10 \mathrm{~g}$ ethanol $(13,14)$.

\section{Statistics}

The comparison between groups was made with Student test for normal distributed data, with ManWhitney test for non-normal distributed data, and Chi-square test for non-parametric data. Spearman test were applied for correlation calculation for non-normal distributed data, and Pearson test for correlation of normal distributed data. $p<0.05$ were considered significant. The correlations were

TABLE 1. Clinical and demographical characteristics of study group compared to control group. The values are expressed as mean (SD) - for normal distributed data, median (quartiles) for non-normal distributed data, and number of subjects for non-parametric data.

\begin{tabular}{|l|c|c|c|}
\hline Parameter & Group 1 (Control) $\mathrm{n}=50$ & Group 2 (Study) N=65 & P value \\
\hline Age (years) & $43.5(37.5-45)$ & $44(40-45)$ & $\mathrm{NS}$ \\
\hline BMI (Quetelet formula)* & $23.72(23-25)$ & $26.3(25.5-27)$ & $\begin{array}{c}\mathrm{p}<0.01 \\
\mathrm{p}<0.01\end{array}$ \\
\hline TAS (mmHg)* & $122(120-124)$ & $124(120-125)$ & $\mathrm{p}<0.05$ \\
\hline TAD (mmHg)** & & & $\mathrm{p}<0.001$ \\
\hline Glycemia (mg\%)* & $79.8(3.49)$ & $87.23(5.45)$ & $\mathrm{NS}$ \\
\hline Current Smoking status/non-smoking status*** & $90(88-97)$ & $86(80-98)$ & $\mathrm{p}<0.05$ \\
\hline $\begin{array}{l}\text { Alcohol consumption (number of patients with } \\
\text { significant ethylic alcohol consumption) }\end{array}$ & $15 / 35$ & $32 / 33$ & $\mathrm{NS}$ \\
\hline CRP (mg\%)** & $5 / 50$ & $11 / 62$ & $\mathrm{p}<0.0001$ \\
\hline Fibrinogen (mg\%)* & $0.5(0.17)$ & $1(0.29)$ & $\mathrm{NS}$ \\
\hline NOx ( $\mu$ mol/L)* & $202.5(145-165)$ & $255.75(220-285)$ & $\mathrm{P}<0.01$ \\
\hline LDL - CST (mg\%)* & $50.25(45.25-52.75)$ & $32.55(30.25-35.75)$ & $\mathrm{p}<0.001$ \\
\hline HDL - CST (mg\%)** & $110(110-130)$ & $140(125-160)$ & $\mathrm{p}<0.01$ \\
& $40(33.5-45$ & $35.23(30-43.25)$ & $\mathrm{p}<0.05$ \\
\hline TG (mg\%)* & $40(33-45)$ & $35(30-44)$ & $\mathrm{NS}$ \\
\hline
\end{tabular}

*Man-Whitney test, $* *$ t-test, $* * *$ Chi-square test, NS - non-significant 
made between MIDAS score and risk factors (which proved significant difference in study group compared with control group).

\section{RESULTS}

The demographical characteristics of study group compared to control group are presented in Table 1.

\section{Relationship between MIDAS score and BMI, CRP, NOx, lipoproteins, glycemia, and blood pressure, in study group}

Significant monotonic positive relationship was identified for MIDAS score with BMI, and CRP values. A negative monotonic relationship between MMSE and NOx was also demonstrated (see Table 2). No significant relationship was identified between MIDAS score and other parameter in study group patients.

\section{DISCUSSIONS}

The association between dyslipidemia and common migraine was proved to be homogeneous in recent researches. There is a positive association between common migraine and dyslipidemia observed in women with migraine but not in men patients (15), however different connection between migraine specific features and a dyslipidemia cannot be excluded in male population (16). Several studies lead to the conclusion that high levels of triglycerides and cholesterol may be involved in a more complex relationship between migraine, atherosclerotic vessel changes and metabolic syndrome. Triglycerides, total cholesterol, low-density lipoprotein cholesterol may be used in the future as potential predictors for migraine and treatment responsivity. The differences in lipids levels between the study group and control group were statistical significant but there is no clear relationship with clinical features of migraine (17).

A direct relation of cigarette-smoking and common migraine has not been proved, but the prevalence of smoking is higher in migraine patients (18). Even though smoking is unlikely to be an independent factor for recurrent migraine attacks (19) it can posted as a negative lifestyle factor as- sociated to common migraine (20). Furthermore, tobacco exposure may lower the threshold of trigeminal-autonomic reflex and more severe migraine attacks may occur (21). Our study proved a significant higher number of smokers in migraine patients group compared to control group.

Regarding the blood pressure, a correlation has been observed between a high diastolic pressure and migraine. The correlation between systolic blood pressure and migraine is not clear (22). An increase of blood pressure in systole may be a major determinant of small arteries' compliance, suggesting a strong connection between vasospasm mechanisms, observed in migraine that is associated with hypertension (23). Over more, patients with common migraine have an increased risk of developing hypertension (24). Hypertension may increase the frequency and severity of migraine attacks or lead to the transformation of episodic migraine in chronic migraine (25). Our study found significant differences between study group and control group in both diastolic and systolic blood pressure, even the values were in normal limits. It is possible that renin-angiotensin-aldosteron system (RAA) having an important implication in correlation of migraine with hypertension, in some selected cases (26). Increased availability of angiotensin II and a higher converting enzyme activity seem to have a pathogenic role in migraine (27). There is strong evidence that a good control of blood pressure may improve the migraine outcome (28). Further studies are needed for clarify the connection between blood pressure values and common migraine.

An increased BMI reflecting obesity and insulin resistance have found to have a higher prevalence among patients with common migraine (29). Obesity was correlated to migraine attacks frequency, with poor treatments efficiency. The adipose tissue plays an important role in the inflammatory process producing active peptides such as leptine and adiponectine. Leptine is a pro inflammatory adipokine and have proportionate levels to body fat stores. Adiponectine has an anti-inflammatory function but is decreasing in obesity, creating disequilibrium in favor of inflammation (30). Obesity is a pro-inflammatory state in which pain-generating-hormones are produced and released from adipose

TABLE 2. Significant monotonic relationship expressed as Spearman's rank correlation ( $p$ values) in study group

\begin{tabular}{|c|c|c|c|c|c|c|c|c|}
\hline & $\begin{array}{c}\text { MMSE and } \\
\text { BMI }\end{array}$ & $\begin{array}{c}\text { MMSE and } \\
\text { CRP }\end{array}$ & $\begin{array}{c}\text { MMSE and } \\
\text { NOx }\end{array}$ & $\begin{array}{c}\text { MMSE and } \\
\text { LDL-CST }\end{array}$ & $\begin{array}{c}\text { MMSE and } \\
\text { HDL-CST }\end{array}$ & $\begin{array}{c}\text { MMSE and } \\
\text { glycemia }\end{array}$ & $\begin{array}{c}\text { MMSE and } \\
\text { TAS }\end{array}$ & $\begin{array}{c}\text { MMSE and } \\
\text { TAD }\end{array}$ \\
\hline$p$ value & $p<0.05$ & $p<0.05$ & $p<0.05$ & NS & NS & NS & NS & NS \\
\hline
\end{tabular}


cells. Substance P, TNF-alpha, interleukine-6, are molecules involved in pain mechanism and were found also to be increased in migraine (31). Therefore the predisposition of obese individuals for more severe migraine attacks and lower treatment responsivity, has a cumulative pathogenethic factors from two separate independent sources: obesity and migraine with a common background represented by inflammation

The complex mechanisms involved in migraine and their connection to high CRP levels is incompletely understood. The link between migraine and high levels of CRP may be induced by various mechanisms as are leukocyte activation, oxidative stress, inflammatory cytokines produced during, and between migraine attacks. High serum levels of TNF-alpha and IL-1 are common in migraine patients (32). Even though common migraine is characterized by episodic attacks, the underlying inflammatory changes are long lasting or irreversible, leading to permanent dysfunction and destabilization of the neurons (33). Migraine attacks are associated with repeated neurogenic inflammation of cranial arteries and dura mater. Several pro inflammatory cytokines released during migraine attacks are involved in the endothelial inflammatory process predisposing to atherosclerotic disease. These molecules may increase the peripheral resistance in migraine patients (34). These findings suggest that hyperactivity of basal arteries induced by inflammatory cytokines hyper production may lead to vasospasm phenomena seen during the attacks (35). CRP may be used in the future to identify the patients with migraine which have a risk for vascular events (36).

The correlation between CRP as marker of inflammation was demonstrated by Welch et al, suggesting that attacks of migraine may involve repeated sterile vascular inflammation (37). Proinflammatory markers as is CRP were proved to be significantly increased in migraine patients (38). There are other studies that proved that the nitrite concentration in migraineurs was significantly lower than healthy subjects, and L-arginine (a NO donor) may improve the clinical symptoms $(39,40)$.

\section{CONCLUSIONS}

The connection between obesity, inflammatory parameters and migraine may be useful for new prophylaxis potent therapies. The individual personalized therapies according with particular risk factors of each patient may be a chance for better results. Identification and control of risk factors in migraine patients may be particularly important.

11. Stewart W.F., Lipton R.B., Dowson A.J., Sawyer J. Development and testing of the Migraine Disability Assessment (MIDAS) Questionnaire to assess headache-related disability, Neurology, 2001, 56(6 Suppl 1):S20-8.

12. Miranda K.M., Espey M.G., Wink D.A. A rapid, simple spectrophotometric method for simultaneous detection of nitrate and nitrite, Nitric Oxide, 2001, 5:62-71.

13. Martin-Moreno J.M., Harris M.E., Breda J., Møller L., AlfonsoSanchez J.L., Gorgojo L. Enhanced labelling on alcoholic drinks: reviewing the evidence to guide alcohol policy, Eur J Public Health, 2013, 23(6):1082-7.

14. Sacco S., Pistoia F., Degan D., Carolei A. Conventional vascular risk factors: their role in the association between migraine and cardiovascular diseases, Cephalalgia, 2015, 35(2):146-64.

15. Goulart A.C., Santos I.S., Lotufo P.A., Benseñor I.M. Gender aspects of the relationship between migraine and cardiovascular risk factors: A cross-sectional evaluation in the Brazilian Longitudinal Study of Adult Health (ELSA-Brasil). Cephalalgia, 2015: 0333102415570494

16. Winter A.C., Berger K., Buring J.E., Kurth T. Body mass index, migraine, migraine frequency and migraine features in women, Cephalalgia, 2009, 29(2):269-78.

17. Kurth T., Gaziano J.M., Cook N.R., Logroscino G., Diener H.C., Buring J.E. Migraine and risk of cardiovascular disease in women. JAMA, 2006, 296:283-291. 
18. López-Mesonero L., Márquez S., Parra P., Gámez-Leyva G., Muñoz P., Pascual J. Smoking as a precipitating factor for migraine: a survey in medical students, J Headache Pain, 2009, 10(2):101-3.

19. Volans G.N., Castleden C.M. The relationship between smoking and migraine. Postgrad Med J. 1976, 52(604): 80-82.

20. Robberstad L., Dyb G., Hagen K., Stovner L.J., Holmen T.L., Zwart J.A. An unfavorable lifestyle and recurrent headaches among adolescents: the HUNT study. Neurology, 2010, 75(8):712-7.

21. Rozen T.D. A history of cigarette smoking is associated with the development of cranial autonomic symptoms with migraine headaches, Headache, 2011, 51(1):85-91.

22. Hagen K., Strovmer L.J., Vatten L., Holmen J., Zwart J.A., Bovim G. Blood pressure and risk of headache: a prospective study of 22 685 adults in Norway. J Neurol Neurosurg Psychiatry, 2002, 72:463-6.

23. Pauca A.L., O'Rourke M.F., Kon N.D. Prospective evaluation of a method for estimating ascending aortic pressure from the radial artery pressure waveform. Hypertension, 2001, 38(4):932-7.

24. Harandi S.A., Togha M., Sadatnaseri A., Hosseini S.H., Jahromi S.R. Cardiovascular risk factors and migraine without aura: A case-control study, Iran J Neurol, 2013, 12(3):98-101.

25. Mathew N.T. Migraine and hypertension, Cephalalgia, 1999, 19 Suppl 25:17-9.

26. Agostoni E., Aliprandi A. Migraine and hypertension, Neurol Sci, 2008, 29 Suppl 1:S37-9.

27. Schrader H., Stovner L.J., Helde G., Sand T., Bovim G. Prophylactic treatment of migraine with angiotensin converting enzyme inhibitor (lisinopril): randomised, placebo controlled, crossover study, BMJ. 2001, 322(7277):19-22.

28. Prentice D., Heywood J. Migraine and hypertension. Is there a relationship? Aust Fam Physician, 2001, 30(5):461-5.

29. Guldiken B., Guldiken S., Taskiran B., Koc G., Turgut N., Kabayel L., Tugrul A. Migraine in metabolic syndrome, Neurologist, 2009, 15(2):55-8.
30. Stofkova A. Leptin and adiponectin: from energy and metabolic dysbalance to inflammation and autoimmunity, Endocr Regul, 2009, 43(4):157-68.

31. Tepper D.E. Migraine and obesity, Headache, 2013, 53(4):719-20.

32. Yilmaz I.A., Ozge A., Erdal M.E., Edgünlü T.G., Cakmak S.E., Yalin O.O. Cytokine polymorphism in patients with migraine: some suggestive clues of migraine and inflammation, Pain Med, 2010, 11(4):492-7.

33. Gergont A., Kaciński sM., Kwinta P. Proinflammatory cytokines in children with idiopathic headache, Przegl Lek, 2005, 62:1269-75.

34. Nagai T., Tabara Y., Igase M., Nakura J., Miki T., Kohara K. Migraine is associated with enhanced arterial stiffness, Hypertens Res, 2007, 30(7):577-83.

35. D’Amico D., Ferraris A., Leone M., Catania A., Carlin A., Grazzi L., Bussone G. Increased plasma nitrites in migraine and cluster headache patients in interictal period: basal hyperactivity of L-arginine-NO pathway? Cephalalgia, 2002, 22(1):33-6.

36. Vanmolkot F.H., de Hoon J.N. Increased C-reactive protein in young adult patients with migraine, Cephalalgia, 2007, 27(7):843-6.

37. Welch K.M., Brandes A.W., Salerno L., Brandes J.L. High hs-CRP level may be a marker of the proinflammatory state in migraine patients, Headache, 2006, 46(2):197-9.

38. Yilmaz Avci A., Lakadamyali H., Arikan S., Benli U.S., Kilinc M. High sensitivity C-reactive protein and cerebral white matter hyperintensities on magnetic resonance imaging in migraine patients, J Headache Pain, 2015, 16;16(1):9.

39. Heshmat-Ghahdarijani K., Javanmard S.H., Sonbolestan S.A., Saadatnia M., Sonbolestan S.A. Endothelial Function in Patients with Migraine without Aura during the Interictal Period, Int J Prev Med, 2015, (15)6:2.

40. Pretnar-Oblak J. Cerebral endothelial function determined by cerebrovascular reactivity to L-arginine, Biomed Res Int, 2014:601515. 